FACULDADE DE CIÊNCIAS ECONÔMICAS DA UFRGS Análise
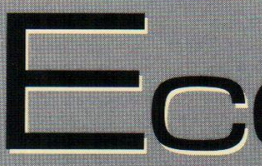

VALORES E PREÇOS DE PRODUÇÃO: UMA RELETURA de MARX Alfredo SAAD FILHO

OS ANTIGOS, OS NOVOS E OS NEO-INSTITUCIONALISTAS: HÁ CONVERGÉ NCIA TEÓ RICA NO PENSAMENTO INSTITUCIONALISTA

Octavio Augusto C. Conceição

A MENSURAÇÃ O DO GRAU DE INDEPENDÉNCIA DO BANCO CENTRAL: UMA ANÁLISE DE SUAS FRAGILIDADES HeLder Ferreira de MENDONÇA

A SUSTENTABILIDADE DA DÍVIDA MOBILIÁ RIA FEDERAL BRASILEIRA: UMA INVESTIGAÇĀ O ADICIONAL VIVIANE LUPORINI

O MODELO dE KRUGMAN EXPLICA A CRISE CAMBIAL. BRASILEIRA EM JANEIRO DE 1999?

Adriano CAMPOS MENEZES E TITO BELCHIOR S. MOREIR/

ESTRANGULAMENTO DA díVIDA EXTERNA E CRESCIMENTO ECONO MICO NA AMÉRICA LATINA: LIÇÓ ES DA DÉCADA DE 80

Marco Vaies buratto E SABino da SiLva P. JR.

CriaÇÃo e desvio de COMÉrCio: ANÁlise do FLuXo COMERCial ENTRE O Brasil. e O MERCOSUl para Alguns PRODUTOS INDUSTRIALIZADOS

Clá Udio Roberto fó fFano Vasconcelos

A EVOLUCÁ̃ DA INDÚ STRIA BRASILEIRA DE CELULOSE E SUA ATUAÇÃO NO MERCADO MUNDIAL

Emerson Martins HILGemberg e Carlos josé Caetano BACHA

A PROTEÇÃo SOCIAL NO PRIMEIRO QUARTEL DO SÉCULO XXI: BRINCANDO COM CENÁRIOS

rosa Maria Marques e Áquilas Mendes

ECONOMIA DO CRIME: ELEMENTOS TEÓ RICOS E EVIDÊNCIAS EMPÍRICAS

Gilberto José SChaefer e Pery Francisco Assis Shikida

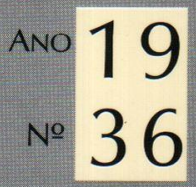


UNIVERSIDADE FEDERAL DO RIO GRANDE DO SUL

Reitora: Profa. Wrana Maria Panizzi

FACULDADE DE CIÊNCIAS ECONÔMICAS

Diretor: Prof. Pedro Cézar Dutra Fonseca

CENTRO DE ESTUDOS E PESQUISAS ECONÔMICAS

Diretor: Prof. Gentil Corazza

DEPARTAMENTO DE CIÊNCIAS ECONÔMICAS

Chefe: Prof. Luiz Alberto Oliveira Ribeiro de Miranda

DAPARTAMENTO DE CIÊNCIAS CONTÁBEIS E ATUARIAIS

Chefe: João Marcos Leão da Rocha

CURSO DE PÓS-GRADUAÇÃO EM ECONOMIA

Coordenador: Prof. Eduardo Pontual Ribeiro

CURSO DE PÓS-GRADUAÇÃO EM ECONOMIA RURAL

Coordenador: Prof. Jalcione Almeida

CONSELHO EDITORIAL: Achyles B. Costa, Aray M. Feldens, Carlos A. Crusius, Carlos G. A. Mielitz Netto, Eduardo A. Maldonado Filho, Eduardo P. Ribeiro, Eugênio Lagemann, Fernando Ferrari Filho, Gentil Corazza, Jan A. Kregel (Univ. of Bologna), Marcelo S. Portugal, Nali J. Souza, Otilia B. K. Carrion, Paulo A. Spohr, Paulo D. Waquil, Pedro C. D. Fonseca, Philip Arestis (Univ of East London), Roberto C. Moraes, Ronald Otto Hillbrecht, Stefano Florissi, Eleutério F. S. Prado (USP), Fernando H. Barbosa (FGV/RJ), Gustavo Franco (PUC/RJ), João R. Sanson (UFSC), Joaquim P. Andrade (UnB), Juan H. Moldau (USP), Paul Davidson (Univ. of Tennessee), Werner Baer (Univ. of Illinois).

COMISSÃO EDITORIAL: Eduardo Augusto Maldonado Filho, Fermando Ferrari Filho, Gentil Corazza, Marcelo Savino Portugal, Paulo Dabdab Waquil; Roberto Camps Moraes.

EDITOR: Fernando Ferrari Filho

EDITOR ADJUNTO: Pedro Silveira Bandeira

SECRETÁRIA: Vanessa Hoffmann de Quadros

REVISÃO DE TEXTOS: Vanete Ricacheski

FUNDADOR: Prof. Antônjo Carlos Santos Rosa

Os materiais publicados na revista Análise Econômica sāo da exclusiva responsabilidade dos autores. É permitida a reproduçāo total ou parcial dos trabalhos, desde que seja citada a fonte. Aceita-se permuta com revistas congêneres. Aceitam-se, também, livros para divulgação, elaboração de resenhas e recensões. Toda correspondência, material para publicação (vide normas na terceira capa), assinaturas e permutas devem ser dirigidos ao seguinte destinatário:

PROF. FERNANDO FERRARI FILHO

Revista Análise Econômica - Av. João Pessoa, 52 CEP 90040-000 PORTO ALEGRE - RS, BRASIL Telefones: (051) 316-3348 e 316-3440 - Fax: (051) 316-3990

E-mail: rae@vortex.ufrgs.br

Análise Econômica

Ano 18, n⿳3 33, março, 2000 - Porto Alegre

Faculdade de Ciências Econômicas, UFRGS, 2000

Periodicidade semestral, março e setembro.

1. Teoria Econômica - Desenvolvimento Regional -

Economia Agrícola - Pesquisa Teórica e Aplicada -

Periódicos. I. Brasil

Faculdade de Ciências Econômicas,

Universidade Federal do Rio Grande do Sul.

CDD 330.05

CDU 33 (81) (05) 


\title{
Criação e desvio de comércio: análise do fluxo comercial entre o Brasil e o Mercosul para alguns produtos industrializados
}

Cláudio Roberto Fóffano Vasconcelos **

\begin{abstract}
Resumo: No presente trabalho objetivou-se analisar, sob a ótica de equilíbrio parcial, os impactos estáticos do processo de integração através da criação e desvio de comércio entre o Brasil e o MERCOSUL, para produtos industrializados selecionados. A conclusão geral do trabalho é a de que há evidências de desvio de comércio somente para as seções VI (produtos das indústrias químicas e conexas) e XVI (máquinas e aparelhos, materiais elétricos). Entretanto, para o conjunto de seçōes da NBM, referente a produtos industrializados considerados na análise, houve uma criação de comércio líquida.
\end{abstract}

Palavras-chave: comércio internacional; criação e desvio de comércio; Mercosul.

Abstract: The aims of study were to analyse, under the partial equilibrium, the static effects of the integration process, through the trade creation and trade diversion between Brazil and the MERCOSUR, for selected industrialised products. A conclusion of this work was that the evidences of the trade diversion are only for sections VI (chemical and related industry products) and XVI (machines and equipment, electrical material). Nevertheless, for the total of the NBM sections, referring to the industrialised products considered in the analysis, there was a liquid trade creation.

Key words: international trade; trade creation and trade diversion; Mercosur.

\section{Introdução}

As discussões acerca dos efeitos do processo de integração dos países do Cone Sul, MERCOSUL, são intensas. Neste contexto, Yeats

\footnotetext{
-O presente trabalho foi organizado a partir do capítulo 4 da tese de doutorado do autor, apresentada ao PIMES/ UFPE.

" Professor Adjunto da Fundação Universidade Federal do Rio Grande. Av. Itália km 8, Campus Carreiros, Rio Grande - RS CEP.96.201-900. Fone/Fax: (53)232-6060. E-mail: crfv@mikrus.com.br.
} 
(1998), analisando os índices de orientação regional e vantagem comparativa revelada para os países do MERCOSUL, para o período entre 1988 e 1994, conclui que a implantação desse acordo de comércio estaria distorcendo o padrão de comércio em termos de eficiência e aproveitamento de vantagem comparativa. Isto é, a reorientação do comércio em favor dos países membros teria ocorrido, em grande parte, em função de produtos intensivos em capital, sendo que estes países não apresentavam um padrão histórico de vantagens comparativas nos setores que mais expandiram suas exportações intrabloco. Portanto, tal situação evidenciaria, segundo o autor, o efeito adverso do desvio de comércio no fluxo intrabloco no MERCOSUL.

Em contraposição a esse argumento de Yeats (1998), pode-se dizer que se a intenção é verificar os efeitos do processo de integração em termos de criação e desvio de comércio, o emprego de uma abordagem direta de mensuração desses impactos poderia vir a contribuir de uma forma mais objetiva para a elucidação da questão de se a implantação do MERCOSUL implicou ou não desvio de comércio superior à criação de comércio.

Desta forma, neste trabalho objetiva-se analisar o fluxo de comércio brasileiro relativo ao MERCOSUL sob a abordagem de Viner (1950) de avaliação dos impactos estáticos do processo de integração através da criação e do desvio de comércio.

Para mensurar a criação e desvio de comércio referente a alguns produtos industrializados da pauta de importações brasileiras provenientes do MERCOSUL ${ }^{1}$, foi empregada, em uma análise de equilíbrio parcial, a abordagem das elasticidades, como proposto em Laird e Yeats (1986).

Além desta seção, o restante deste trabalho é composto pela seção $2^{2}$, onde foram analisados os trabalhos empíricos de mensuração de criação e desvio de comércio, para o caso específico do MERCOSUL. $\mathrm{Na} 3$ foi tratado do método utilizado. Na seção 4 são apresentados os resultados obtidos, e na 5 as considerações finais.

\footnotetext{
' A não inclusão na análise, da criação e desvio de comércio, de bens agrícolas se deve ao fato de que essa análise já foi feita, como no trabalho de Nonnberg e Mendonça (1998). Monteiro e Grenner (1998) e Machado e Cavalcanti (1999).

2 Para a análise teórica do modelo básico de Viner (1950), com as definições de criação e desvio de comércio, e formas de mensuração empírica, ver Vasconcelos (2000, p. 115-132).
} 


\section{Criação e desvio de comércio para a economia brasileira referente ao fluxo de comércio com o Mercosul}

Observa-se com maior freqüência na literatura a utilização das abordagens das elasticidades, do modelo gravitacional e do modelo da taxa de crescimento das importações e taxa de variação do consumo aparente em relação às importações. Da mesma forma, algumas dessas abordagens são encontradas nos trabalhos de mensuração da criação e desvio de comércio para a economia brasileira, referente ao fluxo de comércio com o MERCOSUL.

Com relação à utilização do modelo de crescimento da taxa de importação e taxa de consumo aparente em relação às importações, para a análise do fluxo de comércio entre a economia brasileira e o MERCOSUL, poucos estudos têm sido desenvolvidos. Na literatura consultada, foi encontrado apenas o trabalho de Monteiro e Grenner (1998). A dificuldade de emprego dessa abordagem, como os próprios autores afirmam, está justamente em identificar um país normalizador (ou conjunto de paises normalizadores), visto a necessidade de se isolar as influências de variáveis como renda, preços, câmbio, ganho de produtividade, refletidos nos fluxos de comércio pós-integração.

Monteiro e Grenner analisaram o mercado de trigo brasileiro, para o período entre 1991 e 1995, utilizando dois países (Chile e Peru) e dois conjuntos de países (Peru/Venezuela e América do Sul) como países normalizadores. Os autores concluiram que, para as duas abordagens empregadas (taxa de crescimento das importações e taxa de consumo aparente em relação às importações), houve uma grande criação de comércio na atividade de trigo, e que não houve desvio de comércio líquido no mercado de trigo do Brasil, no período analisado.

Com relação à mensuração da criação e desvio de comércio para o MERCOSUL sob a abordagem das elasticidades, pode-se citar o trabalho de Nonnemberg e Mendonça (1998), que analisaram o impacto da implementação do MERCOSUL no fluxo de comércio de produtos agrícolas brasileiros. Os autores utilizam a metodologia desenvolvida pela UNCTAD e Banco Mundial ${ }^{3}$ para os cálculos dos efeitos mencionados, de acordo com as equações (2) e (3), descritas na seção 3. A

\footnotetext{
${ }^{3}$ Metodologia empregada pelo Software for Market Analysis and Restrictions on Trade (SMART), uma versão simplificada do Trade Policy Simulation Model (TPSM) desenvolvida pela UNCTAD e Banco Mundial.
} 
estimativa da criação e desvio de comércio foi realizada comparandose as médias de três períodos de tempos, 1988/91, 1991/93 e 1994/96, para os produtos trigo, milho, algodão, arroz, carne de bovinos e leite. A conclusão geral a que chegaram foi de que, para todas as hipóteses adotadas (relativas às elasticidades substituição de $-0,5,-1,5$ e -2,5), os valores totais de desvio de comércio foram substancialmente inferiores aos de criação de comércio.

De forma diferente dos dois trabalhos anteriores, Machado e Cavalcanti (1999), considerando os índices de vantagem comparativa revelada e taxa de crescimento das importações intra e extrabloco ${ }^{4}$, investigaram os impactos de criação e desvio de comércio para o fluxo de comércio Brasil-Argentina durante o período de transição (entre a assinatura do tratado em 1991 e a criação da união aduaneira em 1995).

Os resultados obtidos pelos autores são os de que para uma análise agregada, ou seja, o somatório de todos os produtos considerados, o efeito desvio de comércio superou o efeito criação de comércio. De forma desagregada, a um dígito do STIC, tanto no caso das exportações do Brasil para a Argentina quanto no sentido inverso, os resultados evidenciaram que o desvio de comércio no setor de material de transporte representou mais da metade dos impactos estáticos líquidos. Por outro lado, os setores alimentos e animais vivos e gorduras/ceras foram os únicos que apresentaram criação líquida de comércio.

Uma primeira consideração a essa abordagem desenvolvida por Machado e Cavalcanti é a respeito da pressuposição implícita de que o comércio bilateral Brasil-Argentina se realiza apenas sob os preceitos da teoria tradicional do comércio internacional, através das vantagens comparativas. Como mostrado no capítulo 3 em Vasconcelos (2000) e

\footnotetext{
${ }^{4}$ Os autores definiram a mensuração do valor do desvio de comércio para o fluxo de comércio entre o Brasil e a Argentina em duas etapas. A primeira seria a identificação de quais produtos nas exportaçōes argentinas para o Brasil apresentam índice de vantagem comparativa revelada (IVCR) do produto $i$ com valor menor que um, e, concomitantemente, apresenta uma taxa de crescimento das exportaçōes da Argentina para o Brasil acima da taxa de variação de importaçōes extrabloco do Brasil para o produto $i$. A segunda etapa consiste em calcular a diferença entre os valores das importações do Brasil para o produto i procedente da Argentina, para um ano de referência, e o valor das importaçōes "presumidas" do Brasil, supondo que a taxa de crescimento das importaçōes provenientes da Argentina fosse a mesma das importações brasileiras provenientes do resto do mundo. Para a criação de comércio o procedimento é similar, sendo que agora a primeira etapa consiste em identificar os produtos para os quais o IVCR > 1 para os dois países, concomitantemente com a identificaçāo dos produtos cujas exportaçōes da Argentina para o Brasil tenham crescido acima da taxa de variação das importações brasileiras procedente do resto do mundo Na segunda etapa, o procedimento é o mesmo do descrito para o desvio de comércio.
} 
salientado por Brandão et al. (1998, p. 69) e Laird (1997), o aumento nos fluxos de comércio entre o Brasil e a Argentina no tocante a manufaturas intensivas em capital pode estar sugerindo intensificação do comércio intra-industrial.

A segunda consideração é a respeito da mensuração dos valores de desvio e criação de comércio, em que autores utilizam os padrões de importações (ou taxas de importações) extrabloco pré-integração como "normalizador" para as importações intrabloco "presumidas" pósintegração. Pois, como afirma Plummer (1991, p. 173) "a projeção da tendência da taxa de crescimento pré-integração pode acarretar em substancial viés devido, em grande parte, ao diferencial histórico do ambiente econômico dos anos pré-integração relativos aos anos pósintegração".

Outro trabalho que considera os impactos do MERCOSUL sobre a economia brasileira é o de Brandão et al. (1998). Entretanto, os autores analisam os impactos do MERCOSUL sob uma estrutura de modelos de equilíbrio geral aplicados, dessa forma não são calculados a criação e desvio de comércio. Porém, em sua análise, quando tratam do comércio e produção, são mensuradas as mudanças nas exportações e importações brasileiras para vários conjuntos de países (como União Européia, Paises do Pacífico, Argentina, México, América do Norte, América Latina, Resto do Mundo), possibilitando, assim, fazer algumas inferências sobre o desvio de comércio.

Alguns pontos destacados pelos autores são os de que "há evidências de algum desvio de comércio em certos setores", pois se evidenciou que o "aumento das importações de manufaturados intensivos em recursos naturais e em capital da Argentina, da União Européia e dos Países do Pacífico vem acompanhado de queda nas compras oriundas de outras regiōes do continente americano". Para as importaçōes de alimentos, há também indícios de desvio de comércio, uma vez que, "excetuando-se a União Européia e a Argentina, há queda nas importações oriundas de todas as outras regiões" (Brandão et al. 1998, p. 69). Esses últimos resultados, de certa forma, estão em contradição com os resultados de Monteiro e Grenner (1998), Nonnemberg e Mendonça (1998) e Machado e Cavalcanti (1999).

Em sintese, observa-se que, quando se trata dos impactos para a economia brasileira em decorrência da implantação do MERCOSUL, em termos de criação e desvio de comércio, poucos trabalhos são encontrados na literatura. Além disso, apenas Machado e Cavalcanti (1999) consideram, em sua análise, os produtos industrializados. 
Desta forma, o presente capítulo se diferencia dos demais trabalhos descritos nessa seção por analisar especificamente produtos do setor industrial que tenham peso na pauta de importações brasileiras com relação ao conjunto dos demais países pertencentes ao MERCOSUL, empregando uma abordagem de maior utilização na literatura econômica.

\section{Método utilizado}

Neste trabalho foi empregada a abordagem das elasticidades para mensurar a criação e desvio de comércio entre o Brasil e o MERCOSUL para o ano base de 1990. Pois considerou-se o ano de 1990 como aquele imediatamente anterior ao início do funcionamento da primeira etapa de integração, compreendida como formação de uma área de livre comércio.

A utilização desta abordagem decorre, primeiramente, da robustez do modelo de equilibrio parcial do qual a abordagem das elasticidades se origina. Em segundo lugar, a abordagem das elasticidades demanda um volume relativamente pequeno de informações (fluxos de importaçōes, tarifas, elasticidades preço demanda de importações e elasticidade de substituição).

Em resumo, o presente trabalho diferencia-se dos trabalhos anteriores de mensuração da criação e desvio de comércio para a economia brasileira com relação ao MERCOSUL, no sentido de que considera a análise específica de setores industrializados pela abordagem das elasticidades.

A escolha dos setores (definidos em 3.2) deve-se primeiramente em decorrência da argumentação de Yeats (1998) de que haveria evidências de desvio de comércio no fluxo de comércio intraMERCOSUL em alguns setores industrializados. E, de forma secundária, deve-se também ao fato de que as principais atividades do setor agrícola já foram, de certa forma, tratadas na literatura (como nos trabalhos de Monteiro e Grenner (1998); Nonnemberg e Mendonça (1998); Machado e Cavalcanti (1999)).

\subsection{Modelo utilizado 5}

Para a análise do fluxo de comércio entre Brasil e o MERCOSUL, foi utilizado um modelo de equilíbrio parcial na mensuração da criação e desvio de comércio.

\footnotetext{
${ }^{5}$ A descrição algébrica do modelo encontra-se em Vasconcelos (2000, p. 138 -143).
} 
A equação básica do modelo de equilíbrio parcial para a mensuração da criação de comércio (TC) é:

$$
T C=d M_{i j k}=M_{i j k} \cdot \varepsilon_{m} \cdot\left(\frac{d T_{i j k}}{1+T_{i j k}}\right) \cdot \frac{1}{1-\left(\varepsilon_{m} / \eta_{x}\right)}
$$

Onde, $M_{\mathrm{ijk}}$ é o nível inicial de importação do produto i proveniente do país beneficiário $k$; $\varepsilon_{m}$ é a elasticidade-preço demanda de importação para o bem $i$; $\mathrm{dT}_{\mathrm{ijk}}$ representa o corte da tarifa do bem $i ; \mathrm{T}_{\mathrm{ijk}}$ constitui o nível inicial da tarifa para o bem $i$ no país $j$ com relação ao país $k$; e $\eta_{x}$ é a elasticidade oferta de exportação.

Caso se admita que a elasticidade preço da oferta de exportação, com respeito ao preço mundial seja infinita, a expressão (1) se resume em:

$$
T C=d M_{i j k}=M_{i j k} \cdot \varepsilon_{m} \cdot\left(\frac{d T_{i j k}}{1+T_{i j k}}\right)
$$

Para a expressão do desvio de comércio, pode-se utilizar a formulação de Baldwin e Murray (1977) ${ }^{6}$, desde que se aceitem as hipóteses simplificadoras de que a criação de comércio seja igual à variação da produção $(-\Delta v)$, e de que a variação percentual das importações dos países não membros seja igual à variação percentual da produção do país importador.

Outra abordagem é a que considera a elasticidade de substituição entre os produtos provenientes dos países beneficiados com o acordo comercial e os produtos provenientes dos países não beneficiados ${ }^{7}$. Desta forma, a expressão para o desvio de comércio (TD) é:

$$
T D_{i j k}=\frac{M_{i j k}}{\sum M_{i j k}} \cdot \frac{\sum M_{i j k} \cdot \sum M_{i j K} \cdot \varepsilon_{s} \cdot \frac{d\left(P_{i j k} / P_{i j K}\right)}{P_{i j k} / P_{i j K}}}{\sum M_{i j k}+\sum M_{i j K}+\sum M_{i j k} \varepsilon_{s} \cdot \frac{d\left(P_{i j k} / P_{i j K}\right)}{P_{i j k} / P_{i j K}}}
$$

Onde, Mé a importação do produto; $\varepsilon_{s}$ a elasticidade de substitui-

${ }^{6} T D=T C_{i}\left[\frac{M n_{i}}{V_{i}}\right]$, onde $M n_{i}$ é a importação do bem $i$ dos países nāo beneficiados e $V_{i}$ é a produção doméstica. (2).

${ }^{7}$ Empregada pela metodologia da UNCTAD e Banco Mundial na configuração do programa SMART. 
ção; e $\left(\frac{d\left(P_{i j k} / P_{i j K}\right)}{P_{i j k} / P_{i j K}}\right)$ é a mudança nos preços relativos. Os índices $k, K, j$ e i denotam, respectivamente, país membro, país não membro, país doméstico e o bem em questão.

Na equação acima, para a mensuração do desvio de comércio, é necessário conhecer a mudança de preços relativos, $\left(\frac{d\left(P_{i j k} / P_{i j K}\right)}{P_{i j k} / P_{i j K}}\right)$. Segundo Jachia e Teljeur (1999), no caso de uma liberalização preferencial, a qual reduz a tarifa de importação proveniente dos países parcei$\operatorname{ros}\left(T_{\mathrm{ijk}}\right)$ para zero, enquanto mantém uma tarifa positiva nas importações de outras fontes, o preço das importações provenientes dos países parceiros, relativo aos preços de importações de outras fontes, irá cair proporcionalmente à redução da tarifa. Formalmente, a mudança dos preços relativos é descrita por:

$$
\frac{d P R}{P R}=\frac{1+T_{1}^{i j k} / 1+T_{1}^{i j K}}{1+T_{0}^{i j k} / 1+T_{0}^{i j K}}-1
$$

Onde $(d P R / P R)$ é a mudança dos preços relativos; $T$ é a tarifa, os subscritos 0 e 1 são os períodos antes e após a liberação do mercado; e os sobrescritos $k \mathrm{e} K$ denominam países membros e não membros (ou outros países).

De forma semelhante, se o acordo de comércio não acarreta mudanças nas tarifas aplicadas às importações provenientes de países não membros, como é o caso dos acordos de livre comércio, a expressão (4) fica:

$$
\frac{d P R}{P R}=\frac{1+T_{1}^{i j k}}{1+T_{0}^{i j k}}-1
$$

Desta forma, pode-se reescrever a equação (3) empregando essa concepção de mudanças de preços relativos. Assim, tem-se:

$$
T D_{i j k}=\frac{M_{i j k}}{\sum M_{i j k}} \cdot \frac{\sum M_{i j k} \cdot \sum M_{i j K} \cdot \varepsilon_{s} \cdot \frac{d P R}{P R}}{\sum M_{i j k}+\sum M_{i j K}+\sum M_{i j k} \cdot \varepsilon_{s} \cdot \frac{d P R}{P R}}
$$




\subsection{Procedimentos e natureza dos dados para a mensuração da criação e desvio de comércio}

Foram realizados cálculos para a criação e desvio de comércio de acordo com as equações (2) e (6), pressupondo duas situações. Na primeira simulação, adotou-se a hipótese de formação de uma área de livre comércio com tarifa aduaneira zero para todo o fluxo de comércio intrabloco, com início em 1991. Na segunda simulação foi considerado o programa de liberação comercial com seus percentuais de desgravação tarifária com início em 1991, de acordo com o proposto no tratado de constituição do MERCOSUL ${ }^{8}$ (Brasil, 1991).

Os cálculos da criação e desvio de comércio foram realizados em termos de capítulos pertencentes às seguintes seções da nomenclatura brasileira de mercadorias (NBM): seção VI (Produtos das Indústrias Químicas e Conexas) para os capítulos 28 a 38; seção VII (Plástico e Borracha e suas obras) com os capítulos 39 e 40; seção X (Pastas de Madeira, Papel e suas obras) para os capítulos 47 a 49; seção XI (Materiais Têxteis e suas obras) capítulos 50 a 63; seção XV (Metais Comuns e suas obras) capítulos 72 a 83; seção XVI (Máquinas e Aparelhos, Material Elétrico) com os capítulos 84 e 85; seção XVII (Material de Transporte) capítulos 86 a 89 .

A escolha destas seções e seus respectivos capítulos decorre da dúvida lançada, por alguns autores (especificamente, Yeats (1998)), sobre o fluxo de comércio do MERCOSUL ter se caracterizado por apresentar um aumento do comércio intrabloco em produtos de elevada intensidade de capital e de tecnologia. Além disso, considerou-se a importância relativa destas seçōes no valor total importado, como pode ser observado na Tabela 1. Nos anos de 1990 e 1991, as seções selecionadas representaram 35,72\% e 36,45\% das importaçōes brasileiras provenientes do MERCOSUL e 71,33\% e 68,32\%, desconsiderando os valores de importação do setor agrícola (seções I e II).

Inicialmente, para o emprego das equações (2) e (6) de mensuração

\footnotetext{
${ }^{8}$ Não foram consideradas as influências de barreiras não-tarifárias nas relações comerciais entre o Brasil e os demais países do MERCOSUL para as duas formas de simulações descritas acima, bem como as listas de exceções de produtos. Isto porque o primeiro conjunto de simulaçōes, por pressuposiçāo, é realizado para uma situação de pleno funcionamento de uma área de livre comércio, a partir de 1991. Já para o segundo conjunto de simulações, a mensuração e incorporação de barreiras não tarifárias constitui tarefa de dificil realização. Assim, por pressuposição do modelo empregado, as tarifas ad valorem empregadas compreendem os custos de transporte, seguros e incidência de alguma distorçāo não tarifária
} 
da criação e desvio de comércio, são necessárias as informações sobre elasticidades preço de demanda de importação, elasticidade substituição (bens importados de diferentes fontes), tarifas aduaneiras e valores de importação por parte do Brasil provenientes dos países do MERCOSUL.

Com relação às elasticidades preço demanda de importação, foram empregados como referência dois conjuntos de valores. O primeiro conjunjunto utilizado foi das elasticidades calculadas por Carvalho e Parente (1998b), que foram mensuradas, através de cointegração, com dados referentes ao período 1978 a 1996. Considerando a adoção desse período, supõe-se que os resultados dos cálculos das elasticidades devem incorporar as modificações ocorridas na economia brasileira, em decorrência do processo de abertura ocorrido nos anos 90. Entretanto, as elasticidades foram calculadas com base em setores agrupados de acordo com categorias de uso $^{9}$, o que implica alto nível de agregação.

Tabela 1: Participação percentual das seções selecionadas de produtos industrializados na importação total do Brasil proveniente dos países do MERCOSUL, 1990 e 1991

\begin{tabular}{l|c|c|c|c}
\hline & 1990 & & 1991 & \\
\cline { 2 - 6 } \multicolumn{1}{c|}{ Seções da NBM } & $\begin{array}{c}\text { Importação } \\
\text { Total }\end{array}$ & $\begin{array}{c}\text { Importação } \\
\text { Total menos } \\
\text { agricultural }\end{array}$ & $\begin{array}{c}\text { Importação } \\
\text { Total }\end{array}$ & $\begin{array}{c}\text { Importação } \\
\text { Total menos } \\
\text { agricultural }\end{array}$ \\
\hline VI Prod. indústrias químicas e conexas & 9,26 & 18,49 & 7,94 & 14,88 \\
VII Plásticos e borrachas, e suas obras & 3,30 & 6,59 & 3,42 & 6,41 \\
X Pasta de madeira, papel & 2,11 & 4,21 & 1,07 & 2,01 \\
XI Matérias têxteis e suas obras & 10,54 & 21,06 & 10,46 & 19,60 \\
XV Metais comuns e suas obras & 1,70 & 3,40 & 1,86 & 3,48 \\
XVI Máquinas e apar., material elétrico & 6,15 & 12,28 & 6,89 & 12,92 \\
XVII Material de transporte & 2,66 & 5,30 & 4,81 & 9,02 \\
\hline \multicolumn{1}{c}{ TOTAL } & 35,72 & 71,33 & 36,45 & 68,32 \\
\hline
\end{tabular}

Fonte: Secretaria da Receita Federal (1990 e 1991).

1) Por agricultura entende-se como sendo as seçōes I e II (animais vivos e produtos do reino animal e produtos do reino vegetal).

O segundo conjunto de elasticidades preço demanda de importação considerado foi o calculado por Cline e outros (1978), para os Estados Unidos. O emprego dessas elasticidades deve-se ao fato de que elas

${ }^{9}$ Bens de capital, intermediários, não duráveis, duráveis e combustíveis. 
foram mensuradas em nível de seções do Sistema Harmonizado, o que implica maior desagregação em relação ao trabalho de Carvalho e Parente (1998b).

No caso das elasticidades de substituição, como afirmam Carvalho e Parente (1998a), "os ajustes de modelos econométricos para estimar a elasticidade de substituição são pouco confiáveis, além de serem raras as tentativas na literatura neste sentido". Desta forma, utilizou-se como referência para a elasticidade de substituição o valor de $-1,5$, que é usualmente empregado na literatura econômica ${ }^{10}$. Além desse valor; empregaram-se os valores de -0,5 e -2,5 como limite inferior e superior, respectivamente.

Com relação às tarifas de importação para o ano de 1990, foram utilizadas informações contidas em Comércio Exterior do Brasil: Importações (Secretaria da Receita Federal, 1990). Mais especificamente, foram utilizadas as tarifas médias de importação para os respectivos capítulos considerados, obtidas pela divisão entre valor tributável e o valor do imposto calculado. Para as importações provenientes dos países do MERCOSUL, foi descontada destas tarifas a margem de preferência vigente durante o período de transição, de acordo com o Programa de Liberação Comercial proposto no Tratado de Assunção (Brasil, 1991).

Por fim, com relação às informações sobre importação, a publicação Comércio Exterior do Brasil: Importações (Secretaria da Receita Federal, 1990 e 1991) constituiu-se a fonte básica de dados, com valores FOB em dólar americano ${ }^{11}$.

\section{Resultados}

Considerem-se inicialmente os resultados da primeira situação de formação de uma área de livre comércio com o nível de tarifa intrabloco igual a zero para todos os bens, aplicada a partir de 1991 (Tabela 2). Para o conjunto de setores sob análise, os valores da criação de comércio, nas duas hipóteses de elasticidades preço demanda de importação (denominadas nas Tabelas por hipótese A e B), foram maiores que os valores calculados para o desvio de comércio, dadas as hipóteses de

\footnotetext{
${ }^{10} \mathrm{O}$ valor de $-1,5$ para a elasticidade de substituição é considerado também como default no programa SMART da UNCTAD/World Bank (Laird e Yeats, 1986).

${ }^{11}$ Em Vasconcelos (2000), Tabela $1 \mathrm{C}$ do Apêndice C, são encontradas as informações descritas acima e utilizadas para a realização das simulações de criação e desvio de comércio.
} 
elasticidade de substituição $\left(\varepsilon_{s}\right)$ igual a $-0,5$ e -1,5. Para $\varepsilon_{s}$ igual a $-2,5$, o valor do desvio de comércio foi superior ao valor da criação para as duas hipóteses utilizadas de elasticidades preço demanda de importação (Tabela 2).

Tabela 2: Criação e desvio de comércio considerando a situação de formação de uma área de livre comércio plena, ano de 1991

\begin{tabular}{|c|c|c|c|c|c|}
\hline \multirow{2}{*}{ Seções e Capítulos da NBM } & \multicolumn{2}{|c|}{$\begin{array}{c}\text { Criação de comércio } \\
\text { em } 1000 \text { US } \$\end{array}$} & \multicolumn{3}{|c|}{ Desvio de comércio em 1000 US\$ } \\
\hline & $(A)^{*}$ & $(B)^{*}$ & $E_{S}=-0,5$ & $E s=-1,5$ & $E_{s}=-2,5$ \\
\hline \multicolumn{6}{|l|}{ VI - Prod ind químicas e conexas } \\
\hline 28- Produtos químicos inorgânicos & 8962 & 4340 & 1998 & 5913 & 9723 \\
\hline 29- Produtos químicos orgânicos & 18971 & 9187 & 4220 & 12443 & 20388 \\
\hline 30- Produtos farmacêuticos & 339 & 164 & 76 & 226 & 373 \\
\hline 31-Adubos e fertilizantes & 514 & 249 & 115 & 344 & 572 \\
\hline 32 - Extrat. tanantes e tintoriais tintas & 17841 & 8640 & 3952 & 11552 & 18775 \\
\hline 33 - Óleos essenciais e resinóides & 12951 & 6272 & 2849 & 8224 & 13205 \\
\hline 34 - Sabões, ceras artificiais & 3410 & 16.51 & 755 & 2201 & 3571 \\
\hline 35 - Mat. Albuminóide, colas enzimas & 7094 & 3435 & 1574 & 4613 & 7518 \\
\hline 36- Pólvoras, explosivos, fósforos & 59 & 29 & 13 & 38 & 61 \\
\hline 37 - Prods. $p /$ fotografia e cinematog & 5735 & 2777 & 1273 & 3739 & 6104 \\
\hline 38 - Prods Diversos das ind. Químicas & 18198 & 8813 & 4030 & 11775 & 19128 \\
\hline Subtotal & 94074 & 45558 & 20854 & 61069 & 99419 \\
\hline \multicolumn{6}{|l|}{ VII - Plásticos e borracha e suas obras } \\
\hline 39 - Plásticos e suas obras & 19953 & 35562 & 4428 & 12995 & 21198 \\
\hline 40 - Borrachas e suas obras & 17282 & 30802 & 3826 & 11177 & 18151 \\
\hline Subtotal & 37235 & 66364 & 8254 & 24172 & 39349 \\
\hline \multicolumn{6}{|l|}{$X$ - Pasta de madeira, papel } \\
\hline 47 - Pasta de mad, outras mat fibrosas & 429 & 309 & 96 & 288 & 479 \\
\hline 48 - Papel e cartão e suas obras & 4701 & 3380 & 1051 & 3131 & 5182 \\
\hline 49 - Livros, jornais, produtos gráficos & 702 & 505 & 157 & 469 & 778 \\
\hline Subtotal & 5833 & 4193 & 1305 & 3889 & 6439 \\
\hline
\end{tabular}




\begin{tabular}{|c|c|c|c|c|c|}
\hline$X \mid$ - Matérias têxteis e sua obras & & & & & \\
\hline $50-$ Seda & 0 & 0 & 0 & 0 & 0 \\
\hline 51 - Lã, pêlos, fios e tecidos de crina & 5573 & 6761 & 1236 & 3623 & 5902 \\
\hline 52 - Algodão & 19789 & 24008 & 4426 & 13191 & 21841 \\
\hline 53 - Outras fibras têxteis vegetais & 0 & 0 & 0 & 0 & 0 \\
\hline 54 - Filamentos sintét. ou artificiais & 14071 & 17071 & 3122 & 9161 & 14938 \\
\hline 55 - Fibras sintét ou artif. Descont. & 3322 & 4030 & 738 & 2167 & 3538 \\
\hline 56 - Pastas, feltros e falsos tecidos & 1952 & 2368 & 432 & 1257 & 2035 \\
\hline 57 - Tapetes e outs. revest. mat. Têxt. & 968 & 1174 & 213 & 619 & 997 \\
\hline 58 - Tecidos espec., rendas, tapeçaria & 717 & 870 & 158 & 458 & 737 \\
\hline 59 - Tecidos impregnados, revestidos & 1019 & 1236 & 225 & 656 & 1062 \\
\hline 60 - Tecidos de malha & 1357 & 1646 & 299 & 869 & 1403 \\
\hline 61 - Vestuário e seus acess. de malha & 5577 & 6765 & 1228 & 3548 & 5704 \\
\hline 62 - Veste seus acess., exceto malho & 18362 & 22276 & 4045 & 11700 & 18828 \\
\hline 63 - Outs. Artefatos Têxteis confecc. & 2917 & 3539 & 643 & 1864 & 3005 \\
\hline Subtotal & 75623 & 91745 & 16766 & 49111 & 79989 \\
\hline XV - Metais comuns e suas obras & & & & & \\
\hline 72 - Ferro fundido, ferro e aço & 4431 & 4402 & 986 & 2911 & 4776 \\
\hline 73. Obras de ferro fundido, ferro aço & 2725 & 2708 & 605 & 1779 & 2907 \\
\hline 74 . Cobre e suas obras & 187 & 186 & 42 & 124 & 206 \\
\hline 75 - Níquel e suas obras & 0 & 0 & 0 & 0 & 0 \\
\hline 76 - Alumínio e suas obras & 1293 & 1284 & 289 & 859 & 1421 \\
\hline 78 - Chumbo e suas obras & 8 & 8 & 2 & 5 & 9 \\
\hline 79. Zinco e suas obras & 9 & 8 & 2 & 6 & 9 \\
\hline 80 - Estanho e suas obras & 0 & 0 & 0 & 0 & 0 \\
\hline 81 - Outros metais comuns & 44 & 44 & 10 & 30 & 49 \\
\hline 82 - Ferramentas, artefatos cutelaria & 6526 & 6483 & 1438 & 4162 & 6702 \\
\hline 83 - Obras diversas de metais comuns & 562 & 558 & 124 & 362 & 588 \\
\hline Subtotal & 15784 & 15681 & 3498 & 10240 & 16665 \\
\hline
\end{tabular}




\begin{tabular}{|c|c|c|c|c|c|}
\hline \multirow{2}{*}{ Seções e Capítulos da NBM } & \multicolumn{2}{|c|}{$\begin{array}{c}\text { Criação de comércio } \\
\text { em } 1000 \text { US } \$\end{array}$} & \multicolumn{3}{|c|}{ Desvio de comércio em 1000 US\$ } \\
\hline & $\mid A)^{*}$ & $(B)^{*}$ & $E_{s}=-0,5$ & $E_{s}=-1,5$ & $E_{s}=-2,5$ \\
\hline \multicolumn{6}{|l|}{ XVI - Máq. e apar., material elétrico } \\
\hline 84- Reator nucl. Maq Inst. Mecânic. & 57260 & 26260 & 13406 & 39273 & 63954 \\
\hline 85 - Máquinas Apar, Mats. Elétricos & 9353 & 4289 & 2185 & 6375 & 10342 \\
\hline Subtotal & 66612 & 30550 & 15591 & 45648 & 74296 \\
\hline \multicolumn{6}{|l|}{ XVII - Material de transporte } \\
\hline 86-Veículos material $p /$ vias férreas & 0 & 0 & 0 & 0 & 0 \\
\hline 87 - Veículos autom., tratores, ciclo & 27536 & 36724 & 6429 & 18739 & 30365 \\
\hline 88 - Aeronave, out. op. Aéreos partes & 706 & 942 & 167 & 500 & 830 \\
\hline 89 - Embarcaç. E estruturas flutuantes & 154 & 205 & 36 & 104 & 167 \\
\hline Subtotal & 28396 & 37871 & 6632 & 19342 & 31362 \\
\hline SOMA & 323557 & 291962 & 72900 & 213471 & 347519 \\
\hline
\end{tabular}

Fonte: Vasconcelos (2000); cálculos do trabalho.

${ }^{\star}$ Hipótese A: utiliza as elasticidades contidas em Carvalho e Parente (1998b);

*Hipótese (B): utiliza as elasticidades de Cline e outros (1978)

Nesta análise agregada do conjunto de setores, a criação de comércio por parte da economia brasileira, ou seja, o aumento da importação proveniente do MERCOSUL, deslocando a produção doméstica, estaria entre $14,3 \%$ e $12,9 \%$ da importação efetivamente ocorrida no ano de $1991^{12}$. Da mesma forma, considerando as elasticidades de substituição de $-0,5$, 1,5 e -2,5, o desvio de comércio estimado, ou seja, a substituição de importação de terceiros países por parte do Brasil em favor dos países membros estaria entre 3,2\%,9,4\% e 15,3\% da importação efetivamente ocorrida no ano de 1991, para as respectivas elasticidades de substituição.

Portanto, nesta primeira simulação, em termos agregados, os resultados evidenciam que não haveria uma discrepância na diferença entre os valores de criação e desvio de comércio para os setores de produtos industrializados considerados na análise.

Em termos desagregados, tomando como referência para o desvio de comércio a elasticidade de substituição igual a -1,5, percebe-se que, para todos os capítulos da seção VI (produtos das indústrias quí-

${ }^{12}$ Considerando que a importaçāo total do Brasil proveniente do MERCOSUL foi em 1991 de US\$ 2268370 mil, em valor FOB e preços correntes. 
micas e conexas), a criação de comércio foi maior que o desvio sob a hipótese de elasticidade preço demanda de importação $\left(\varepsilon_{m}\right)$ igual a 2,003 (hipótese A). Por outro lado, admitindo $\varepsilon_{m}=-0,97$ (hipótese B), os valores da criação de comércio se tornam inferiores ao de desvio de comércio para toda a seção VI, no caso da elasticidade substituição ser igual a -1,5 (Tabela 2).

Da mesma forma, a seção XVI (máquinas e aparelhos, material elétrico) apresentou valores de criação de comércio superior ao desvio de comércio para as elasticidades da hipótese $\mathrm{A}\left(\varepsilon_{m}=-1,897\right)$ e elasticidade substituição igual a $-1,5$. Esses resultados se invertem para o emprego da hipótese $\mathrm{B}\left(\varepsilon_{m}=-0,87\right)$.

Para as seções VII (plásticos e borracha e suas obras), X (pasta de madeira e papel), XI (matérias têxteis e suas obras), XV (metais comuns e suas obras) e XVII (material de transporte) caso se admita uma elasticidade substituição igual a -1,5, os valores da criação de comércio foram superiores aos valores do desvio de comércio para as duas hipóteses ( $\mathrm{A}$ e B) de elasticidades preços de demanda utilizadas (Tabela 2).

Os resultados evidenciaram que, para a situação hipotética de pleno funcionamento de uma área de livre comércio em 1991 entre o Brasil e os demais países do MERCOSUL, nenhum dos capítulos das seções VII, X, XI, XV e XVII indica desvio de comércio superior à criação de comércio, no caso da adoção da elasticidade de substituição igual a -1,5 e elasticidade preço demanda de importação em torno de 2 (hipótese A da Tabela 2). Portanto, nessa simulação somente as seções, nas quais se utilizou elasticidade preço demanda de importação com valores menores que um (como nas seções VI e XVI na hipótese B da Tabela 2), apresentam valores de criação de comércio inferiores aos de desvio de comércio.

Por outro lado, no caso de uma elasticidade substituição igual a 2,5, os resultados se invertem, ou seja, a criação de comércio é inferior ao valor do desvio de comércio, no caso da adoção do conjunto de elasticidades da hipótese A. Neste caso, somente para valores elevados de elasticidades preço demanda de importação, como os utilizados na hipótese B para as seções VII $\left(\varepsilon_{m}=-3,57\right)$, XI $\left(\varepsilon_{m}=-2,43\right)$ e XVII $\left(\varepsilon_{m}=\right.$ -2,53), é que os valores de criação de comércio tornam-se superiores aos de desvio de comércio.

Por fim, considerando o limite inferior da elasticidade de substituição (ou seja, - 0,5 ), todas as seções selecionadas apresentaram valores de criação de comércio superiores aos do desvio para as duas hipóteses de elasticidade preços demanda de importação utilizadas. 
Considerando agora o programa de liberação comercial para os anos de transição entre 1991 e 1994, a simulação realizada admitiu um corte linear de tarifas intrabloco no valor de $47 \%^{13}$ para o ano de 1991 . Os resultados de criação e desvio de comércio para essa situação estão na Tabela 3.

Como se pode verificar, para o conjunto dos capítulos analisados, a criação de comércio seria em torno de 6,7\% e 6,0\% da importação brasileira proveniente do MERCOSUL referente ao ano de 1991, para as respectivas hipóteses $\mathrm{A}$ e $\mathrm{B}$ de elasticidade preço demanda de importação utilizadas, e o desvio de comércio entre $1,5 \%, 4,5 \%$ e 7,4\%, para as elasticidades de substituição de -0,5, -1,5 e -2,5. Assim, de forma agregada, a formação de uma área de livre comércio nos termos do Tratado de Assunção implica que, para o ano de 1991, o desvio de comércio não excederia a criação de comércio, no caso da elasticidade substituição igual a -1,5.

Para a hipótese de elasticidade substituição de -1,5, as seções VI (produtos das indústrias químicas e conexas) e XVI (máquinas e aparelhos, material elétrico) apresentaram desvio de comércio superior ao de criação de comércio, isto no caso de se empregar a elasticidade preço demanda de importação da hipótese B, ou seja, $\varepsilon_{m}=-0,97$ e $\varepsilon_{m}=-0,87$, para as seções VI e XVI, respectivamente (Tabela 3).

Por outro lado, para a elasticidade substituição igual a $-2,5$, as seções VI, X, XV e XVI apresentaram valores de desvio de comércio superiores aos de criação, para qualquer uma das duas hipóteses (A ou B) de elasticidade preço demanda de importação utilizadas (Tabela 3). Somente para elevados valores de elasticidades preço demanda de importação, como os utilizados na hipótese B para as seções VII $\left(\varepsilon_{m}=-3,57\right), \mathrm{XI}\left(\varepsilon_{m}=-2,43\right)$ e XVII $\left(\varepsilon_{m}=-2,53\right)$, é que os valores de criação de comércio tornam-se superiores aos de desvio de comércio.

Considerando a elasticidade de substituição igual a $-0,5$, todas as seções selecionadas apresentaram valores de criação de comércio superiores aos do desvio, para as duas hipóteses de elasticidades preços demanda de importação utilizadas (Tabela 3).

Percebe-se, então, que estes resultados são idênticos aos anteriormente descritos para uma situação de funcionamento imediato da área de livre comércio (Tabela 2). Portanto, para os capítulos considerados nessa análise, os resultados evidenciaram que os capítulos 28 a 38 da seção VI (produtos das indústrias químicas e conexas) e capítulos 84 e 85 da seção

\footnotetext{
${ }^{13}$ De acordo com o Artigo Terceiro do Anexo I (Programa de liberação comercial) do Tratado de Assunção (Brasil, 1991)
} 
XVI (máquinas e aparelhos, material elétrico, etc.) foram os que apresentaram maior evidência de desvio de comércio superior à criação de comércio.

Tabela 3: Criação e desvio de comércio considerando a situação de formação de uma área de livre comércio com restrições, ano de 1991

\begin{tabular}{|c|c|c|c|c|c|}
\hline \multirow{2}{*}{ Seções e Capítulos da NBM } & \multicolumn{2}{|c|}{$\begin{array}{l}\text { Criação de comércio } \\
\text { en } 1000 \text { US\$ }\end{array}$} & \multicolumn{3}{|c|}{ Desvio de comércio em 1000 US $\$$} \\
\hline & $(A)^{*}$ & $(B)^{*}$ & $E_{s}=-0.5$ & $E_{s}=-1.5$ & $E_{s}=-2,5$ \\
\hline \multicolumn{6}{|l|}{ VI - Prod ind químicas e conexas } \\
\hline 28 - Produtos químicos inorgânicos & 4212 & 2040 & 942 & 2809 & 4652 \\
\hline 29 - Produtos químicos orgânicos & 8917 & 4318 & 1993 & 5930 & 9802 \\
\hline 30 - Produtos farmacêuticos & 159 & 77 & 36 & 107 & 177 \\
\hline 31 - Adubos e fertilizantes & 242 & 117 & 54 & 162 & 270 \\
\hline 32 - Extrat. tanantes e tintoriais tintas & 8385 & 4061 & 1870 & 5542 & 9125 \\
\hline 33 - Óleos essenciais e resinóides & 6087 & 2948 & 1353 & 3985 & 6522 \\
\hline 34 - Sabões, ceras artificiais & 1603 & 776 & 357 & 1058 & 1740 \\
\hline 35 - Mat. Albuminóide, colas enzimas & 3334 & 1615 & 744 & 2208 & 3641 \\
\hline 36 - Pólvoras, explosivos, fósforos & 28 & 13 & 6 & 18 & 30 \\
\hline 37 - Prods $\mathrm{p} /$ fotografia e cinematog. & 2696 & 1305 & 602 & 1787 & 2949 \\
\hline 38 - Prods. Diversos das ind Químicas & 8553 & 4142 & 1907 & 5651 & 9302 \\
\hline Subtotal & 44215 & 21412 & 9866 & 29257 & 48211 \\
\hline \multicolumn{6}{|l|}{ VII - Plásticos e borracha e suas obras } \\
\hline 39 - Plásticos e suas obras & 9378 & 16714 & 2094 & 6215 & 10252 \\
\hline 40 - Borrachas e suas obras & 8123 & 14477 & 1811 & 5365 & 8831 \\
\hline Subtotal & 17500 & 31191 & 3905 & 11581 & 19083 \\
\hline \multicolumn{6}{|l|}{$X$ - Pasta de madeira, papel } \\
\hline 47. Pasta de mad, outras mat fibrosas & 202 & 145 & 45 & 136 & 226 \\
\hline 48 - Papel e cartão e suas obras & 2210 & 1589 & 495 & 1480 & 2459 \\
\hline 49 - Livros, jornais, produtos gráficos & 330 & 237 & 74 & 221 & 368 \\
\hline Subtotal & 2742 & 1971 & 614 & 1837 & 3053 \\
\hline
\end{tabular}




\begin{tabular}{|c|c|c|c|c|c|}
\hline \multirow{2}{*}{ Seções e Capítulos da NBM } & \multicolumn{2}{|c|}{$\begin{array}{l}\text { Criação de comércio } \\
\text { em } 1000 \text { US } \$\end{array}$} & \multicolumn{3}{|c|}{ Desvio de comércio em 1000 US $\$$} \\
\hline & $|A|^{*}$ & $(B)^{*}$ & $E_{s}=-0.5$ & $E_{5}=-1.5$ & $E_{s}=-2.5$ \\
\hline $\mathrm{XI}$ - Matérias têxteis e sua obras & & & & & \\
\hline $50 \cdot$ Seda & 0 & 0 & 0 & 0 & 0 \\
\hline 51 - Lã, pêlos, fios e tecidos de crina & 2619 & 3178 & 585 & 1734 & 2859 \\
\hline 52 - Algodão & 9301 & 11284 & 2084 & 6233 & 10356 \\
\hline 53 - Outras fibras têxteis vegetais & 0 & 0 & 0 & 0 & 0 \\
\hline 54 - Filamentos sintét. ou artificiais & 6614 & 8023 & 1476 & 4382 & 7228 \\
\hline 55 - Fibras sintét ou artif. Descont. & 1561 & 189.4 & 349 & 1036 & 1709 \\
\hline 56- Pastas, feltros e falsos tecidos & 918 & 1113 & 204 & 605 & 994 \\
\hline 57. Tapetes e outs. revest. mat. Têxt. & 455 & 552 & 101 & 299 & 490 \\
\hline 58 - Tecidos espec., rendas tapeçaria & 337 & 409 & 75 & 221 & 363 \\
\hline 59- Tecidos impregnados, revestidos & 479 & 581 & 107 & 316 & 519 \\
\hline 60 - Teridos de malha & 638 & 774 & 142 & 419 & 688 \\
\hline 61 - Vestuário e seus acess. de malha & 2621 & 3180 & 583 & 1718 & 2813 \\
\hline 62 . Vest.e seus acess exceto malha & 8630 & 10470 & 1920 & 5660 & 9273 \\
\hline 63. Outs. Artefatos Têxteis confecc. & 1371 & 1663 & 305 & 900 & 1476 \\
\hline Subtotal & 35543 & 43120 & 7931 & 23522 & 38766 \\
\hline XV - Metais comuns e suas obras & & & & & \\
\hline 72 . Ferro fundido, ferro e aço & 2082 & 2069 & 466 & 1386 & 2293 \\
\hline 73- Obras de ferro fundido, ferro aço & 1281 & 1273 & 286 & 850 & 1403 \\
\hline 74 - Cobre e suas obras & 88 & 87 & 20 & 59 & 98 \\
\hline 75 - Níquel e suas obras & 0 & 0 & 0 & 0 & 0 \\
\hline 76 - Alumínio e suas obras & 608 & 604 & 136 & 407 & 675 \\
\hline
\end{tabular}

Continua... 


\begin{tabular}{l|ccccc}
\hline 78 - Chumbo e suas obras & 4 & 4 & 1 & 2 & 4 \\
79- Zinco e suas obras & 4 & 4 & 1 & 3 & 4 \\
80 - Estanho e suas obras & 0 & 0 & 0 & 0 & 0 \\
81 - Outros metais comuns & 21 & 21 & 5 & 14 & 23 \\
82 - Ferramentas, artefatos cutelaria & 3067 & 3047 & 682 & 2012 & 3298 \\
83 - Obras diversas de metais comuns & 264 & 262 & 59 & 174 & 286 \\
Subtotal & 7418 & 7370 & 1655 & 4907 & 8085 \\
XVI- Máq. e apar., material elétrico & & & & & \\
84 - Reator nucl. Maq. Inst. Mecânic. & 26912 & 12342 & 6341 & 18809 & 31000 \\
85 - Máquinas Apar, Mats. Eléfricos & 4396 & 2016 & 1035 & 3063 & 5039 \\
$\quad$ Subtotal & 31308 & 14358 & 7376 & 21873 & 36039 \\
XVII - Material de transporte & & & & & \\
86 - Veículos material p/vias férreas & 0 & 0 & 0 & 0 & 0 \\
87 - Veículos autom., tratores, ciclo & 12942 & 17260 & 3046 & 9011 & 14816 \\
88 - Aeronave, out. ap. Aéreos partes & 332 & 443 & 79 & 235 & 392 \\
89 - Embarc. e estruturas flutuantes & 72 & 97 & 17 & 50 & 82 \\
$\quad$ Subtotal & 13346 & 17799 & 3141 & 9297 & 15290 \\
\hline \multicolumn{1}{c}{ SOMA } & 152072 & 137222 & 34488 & 102274 & 168526 \\
\hline
\end{tabular}

Fonte: Vasconcelos (2000); cálculos do trabalho

* Hipótese (A): utiliza as elasticidades contidas em Carvalho e Parente (1998b);

* Hipótese (B): utiliza as elasticidades de Cline e outros (1978).

\section{Considerações finais}

Um primeiro aspecto a ressaltar é o de que os valores encontrados, tanto para a criação quanto para o desvio de comércio, são valores potenciais, não estando assim diretamente relacionados com as modificações ocorridas nas importações no ano de 1991. Isto porque os valores encontrados dependem diretamente dos valores das importações correntes de um ano base, das elasticidades preço demanda de importação, da elasticidade de substituição e da variação das tarifas aduaneiras.

Com relação às simulações feitas para o ano de 1991, referentes à etapa de implementação de uma área de livre comércio no MERCOSUL, evidenciou-se que, para o conjunto das sete seçōes da NBM selecionadas, o valor da criação de comércio parece superior ao valor do desvio de comércio, no caso de se adotar a elasticidade substituição de -1,5. Logo, esses resultados não confirmaram o efeito adverso de desvio de comércio na implementação do MERCOSUL, com relação à economia brasileira, no caso específico de alguns produtos industrializados relativamente intensivos em capital.

Em termos desagregados, observou-se que, das seções da NBM selecionadas, somente a seção VI (produtos das indústrias químicas e 
conexas) e a seção XVI (máquinas e aparelhos, material elétrico, etc.) foram as que apresentaram maior evidência de desvio de comércio superior à criação de comércio, ou de desvio de comércio líquido.

Outro fato importante a se ressaltar é que o desvio de comércio esperado para a seção XVII (material de transporte) não se verificou nas simulações realizadas. Isto pode ser devido, em parte, à utilização de elasticidades preço demanda de importação muito altas nas duas hipóteses feitas $\left(\varepsilon_{m}=-1,897\right.$ e -2,53), acarretando valores elevados para a criação de comércio. Como foi verificado para outras seções, o emprego de elasticidade preço demanda com valores inelásticos acarretou valores da criação de comércio inferiores aos de desvio de comércio, no caso do emprego de elasticidade substituição igual a -1,5.

Portanto, pode-se inferir que os resultados encontrados com relação ao fluxo de comércio brasileiro com destino ao MERCOSUL, parcialmente corroboram os resultados de Yeats (1998) e de Brandão et al. (1998, p. 69), pois há evidência de desvio de comércio, e conseqüente redução de bem-estar, para os capítulos pertencentes às seções VI e XVI.

Entretanto, em relação ao trabalho de Machado e Cavalcanti (1999), os resultados obtidos no presente capítulo divergem. Pois, neste estudo, os autores encontraram um desvio de comércio líquido para toda a pauta de comércio (a um dígito do STIC) entre Brasil e Argentina, referente ao período 1991/92. Como já salientado na seção 3.2, estes resultados podem estar refletindo algum viés, ou seja, uma superestimação do desvio de comércio, em decorrência da abordagem utilizada pelos autores.

Por fim, a análise dos efeitos de criação e desvio de comércio no âmbito do processo de integração comercial entre o Brasil e os países Argentina, Paraguai e Uruguai, referente a alguns produtos industrializados, caracterizou-se pela predominância do efeito de criação de comércio.

\section{Referências bibliográficas}

BALDWIN, R. E.; MURRAY, T. (1977) "NFM tariff reduction and developing country trade benefits under the GSP". The Economic Journal. $n^{\circ}$ 87, March, p. 30-46.

BRANDĀO, A. S. (1998) "Uma análise quantitativa dos impactos do Mercosul sobre o Brasil". In: BRANDÃO, A. S.; PEREIRA, L. V. (organizadores) MERCOSUL: perspectivas da integração. $3^{\mathrm{a}}$ ed., Rio de Janeiro: Fundação Getúlio Vargas, p. 47-74. 
BRASIL. (1991) "Decreto n. 350, de 21 de novembro de 1991. Promulga o Tratado para a Constituição de um Mercado Comum entre a República Argentina, a República Federativa do Brasil, a República do Paraguai e a República Oriental do Uruguai". Diário Oficial da República Federativa do Brasil, Brasilia, nº 226, Seção 1, p. 26443, 22 nov. 1991.

CARVALHO, A.; PARENTE, M. A. (1999) Impactos da integração da área de Liure Comércio das Américas. Brasilia: IPEA (Texto para discussão n 635, março).

. (1998a) "Impactos da integração comercial: Brasil - Estados Unidos”. Revista Econômica do Nordeste, V. 29, número especial, julho, p. 845-896.

. (1998b) "Estimação de equações de demanda de importações por categoria de uso para o Brasil (1978/1996)”. In. Anais. Vitória: ANPEC, p. 262-282.

CLINE, R.W.; KAWANABE, N.; KRONSJO. T. O. M. (1978) Trade negotiations in the Tokyo round: a quantitative assessment. Washington: The Brookings Institution. Apud CARVALHO, A.; PARENTE, M. A. (1998b) op. cit.

JACHIA, L.; TELJEUR, E. (1999) Free trade between South Africa and the European Union: a quantitative analysis. UNCTAD Discussion Papers $n^{\circ}$ 141, May, Geneva.

LAIRD, S. (1997) Mercosur: objectives and Achievements. Word Trade Organization (Staff Working Paper TPRD9702), June.

LAIRD, S. e YEATS, A. (1986). The UNCTAD trade policy simulation model: a note on the methodology, data and uses. UNCTAD, Discussion Papers $n^{\circ} 19$, Geneva.

MACHADO, J. B. M.; CAVALCANTI, M. A (1999) "Determinantes do comércio bilateral Argentina-Brasil: uma avaliação dos impactos estáticos do processo de integração no Mercosul". In: Anais. Belém: ANPEC. (em cd-room).

MONTEIRO, O. da SILVA; GRENNER, T. (1998) "Wheat en Mercosur is there any trade: divertion?” In: Anais, Brasilia: SOBER, V. 1, p.785-801. NONNENBERG, M. J. B; MENDONÇA, M. J. C. (1998) "Criação e desvio de comércio no Mercosul: o caso dos produtos agrícolas". In. Anais. Vitória: ANPEC, p. 947-961. 
PLUMMER, M. G. (1991) "Ex-post empirical estimates of the second enlargement: the case of Greece”. Weltwirtschaftliches Archiv. V.127, p. 171-182.

SECRETARIA DA RECEITA FEDERAL. (1990) "Comércio exterior do Brasil: importação”. Brasília: Receita Federal, Ano 19. V. III.

. (1991) "Comércio exterior do Brasil: importação". Brasília: Receita Federal, Ano 20. V. III.

VASCONCELOS, C. R. F. (2000) O comércio Brasil-Mercosul na década de 90: uma análise pelas óticas da intensidade fatorial, comércio intraindústria e criação e desvio de comércio. Recife: Universidade Federal de Pernambuco (Tese de Doutorado) 221p.

VINER, J. (1950) The customs union issue. New York: Carnegie endowment for international peace. $221 \mathrm{p}$.

YEATS, A. (1998) "Does Mercosur's trade performance raise concerns about the effects of regional trade arrangements?" The World Bank Economic Review, V. 12, n 1, p. 1-28. 